\title{
Impact of insecticide-treated nets and indoor residual spraying on malaria case prevalence in Geita and Nyang'hwale districts of Tanzania
}

\author{
Kiputa Thobias Gaudence ${ }^{1,2 *}$, Roy Mwenechanya ${ }^{1}$ and Kennedy Choongo ${ }^{1}$ \\ ${ }^{1}$ Department of Biomedical Sciences, School of Veterinary Medicine, University of Zambia, P. O. Box 32379, \\ Lusaka, ZAMBIA \\ ${ }^{2}$ Department of Livestock and Fisheries, Geita District Council, P.O.Box 83, Geita, TANZANIA \\ *Corresponding author. E-mail: kiputatobias@yahoo.com
}

Received: August 21, 2016; Revised received: October 20, 2017; Accepted: January 31, 2018

\begin{abstract}
Malaria is a protozoan disease and one of the leading causes of illness and deaths in the world. Malaria is predominant in the tropics and subtropics, and it is reported that malaria kills a child every minute. In Tanzania at least $40 \%$ of outpatient attendances are attributable to malaria. This study assessed the impact of Insecticidetreated nets (ITNs) + indoor residual spraying (IRS) in Geita district, IRS alone in Nyang'hwale district and compared the two interventions between the two districts of Tanzania. In a retrospective cross-sectional study, district malaria surveillance data for five years (2011-2015) and two years (2013-2014) were collected and analyzed for Geita and Nyang'hwale districts respectively. A total of 1,387,805 ITNs were distributed and 435,719 households sprayed between 2011 and 2015, however IRS coverage was uneven. There was evidence of malaria prevalence reduction from $53 \%$ to $12 \%$ in Geita district within the five years of intervention. The ITNs coverage was associated with a reduction in malaria prevalence while IRS was not. In Nyang'hwale district malaria cases increased from 103,788 to 123,337 cases in 2013 and 2014 respectively, and were accompanied by decreased households sprayed from 49,554 to 41,632 . Furthermore, only ITNs had a significant effect in the combination. However, even at $100 \%$ ITNs coverage, the estimated probability of finding malaria cases would not be zero. Therefore, based on this study, the use of the two interventions that both use pyrethroids in combination has insignificant benefits and should be avoided unless IRS insecticide is not a pyrethroid.
\end{abstract}

Keywords: Geita and Nyang'hwale districts, Insecticide-treated nets, Indoor residual spraying, Tanzania

\section{INTRODUCTION}

Parasites of the genus Plasmodium cause malaria in humans, and the highest population at risk of malaria is in Africa (WHO, 2015). Based on WHO. (2016) report 3.2 billion people are at risk of suffering from malaria worldwide. According to Moyer et al. (2012), malaria has been with humans for at least 50000 years and the particularly dangerous species, Plasmodium falciparum, has been afflicting Africa for the past 6000 years. Malaria occurs almost exclusively in the tropics and subtropics and approximately, $40 \%$ of the world's population, mostly those living in the world's poorest countries, are at risk of malaria (Okonko et al., 2009). Despite progress in reducing the malaria burden over the past half-decade, malaria remains a leading cause of mortality and morbidity in the developing world (Kim et al., 2012). On Tanzania Mainland, more than $40 \%$ of all outpatient attendances are attributable to malaria, (CDC, 2014). Furthermore, 93\% of the population on the mainland lives in malaria-endemic areas (PMI, 2014). By regions, prevalence is highest in Geita (32\%), Kigoma (26\%), and Lindi (26\%), however, malaria is more common in Mainland Tanzania (10\% prevalence) compared to Zanzibar ( $<1 \%$ prevalence) (United Republic of Tanzania, 2013a). In the countries where malaria is a problem, interventions to prevent and treat the disease are in progress. However, Insecticide Treated Nets are the primary means for malaria prevention worldwide (CDC, 2014). Currently, Insecticide-treated nets (ITNs) + indoor residual spraying (IRS) have proved to be highly effective malaria control interventions being deployed in countries where malaria is endemic to achieve objectives on malaria control and elimination programs (Simon et al., 2013, Lines and Kleinschmidt, 2013).

In Africa, the combination of ITNs and IRS has become a commonplace practice (Okumu and Moore, 2011). These interventions ITNs and IRS are being deployed to achieve World Health Assembly Roll Back Malaria and Millennium Development Targets (Kleinschmidt et al., 2009).

In Tanzania, IRS has been found suitable to combine with ITNs in the districts with a large number of malaria cases (Mboera et al., 2013). Geita and Nyang'hwale districts are among the areas in Tanzania 
where IRS has been implemented (htt://www.rti.org/ files/TVCSP-Success-Geita-Tanzania; PMI, 2014). Research on whether the combined intervention help reduce malaria prevalence has been done in several districts. However, many researches were focused on malaria prevalence based on parasitological indices rather than on malaria cases prevalence (clinical cases and confirmed cases) (Mashauri et al., 2013 and West et al., 2014).

A randomized trial on the impact of IRS and ITNs combined with IRS on malaria prevalence was performed in Mleba district (Kagera) and based on parasitological/haematological indices (parasitaemia/ anaemia) (West et al., 2014 and Mboera et al., 2013). There is no study which has been done to assess the impact of IRS and ITNs on the prevalence of malaria cases (West et al., 2014). No research has been conducted in Geita and Nyang'hwale districts, whether based on parasitological indices or malaria cases, on the impact of IRS and ITNs on malaria prevalence (Nyang'hwale District Health Departments). Therefore, conducting research on the impact of IRS and ITNs on malaria cases in Geita and Nyang'hwale districts, which are within the region with the highest malaria prevalence in Tanzania (32\%) (United Republic of Tanzania, 2013), is of great significance to fill the gap and add knowledge on impact of the combined intervention on malaria cases (clinical cases and confirmed cases) prevalence.

\section{MATERIALS AND METHODS}

Malaria morbidity: Annually aggregated district malaria case data for 2011, 2012, 2013, 2014 and 2015 were obtained from Geita and Nyang'hwale Districts' Health Departments. Malaria cases consisted of both clinically diagnosed cases and microscopically confirmed cases. At health centre level malaria cases are recorded in special forms called Health Information

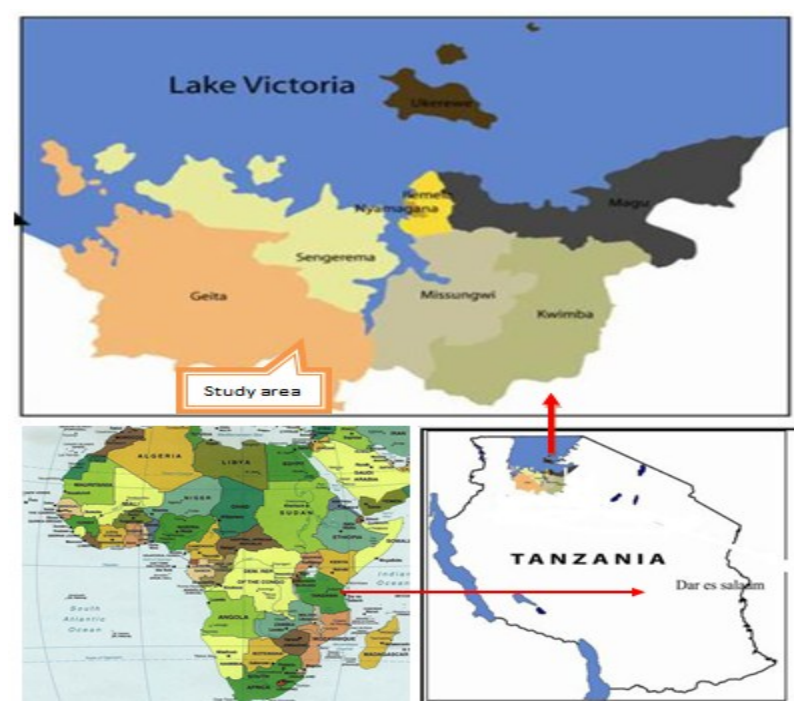

Fig. 1. Map showing the study area, Geita and Nyang'hwale districts.
Management System forms. Data are compiled monthly at health centre level into a report which is submitted to the district hospital each month. At the Districts' Health Departments there are special persons, the Malaria Focal Persons, who compiles malaria cases data for further use.

Population and housing data: Both population and housing data for 2011, 2012, 2013, 2014 and 2015 were obtained from the Districts' Planning, Monitoring and Statistics Departments. The data served as denominators for IRS and ITNs coverage rate calculation. This type of study is deemed not to be human subjects research/did not need human specimen, and patient identifiers were not included in the data (Kamuliwo et al., 2013 and Kigozi et al., 2012), and therefore, didn't need ethical clearance. Geita and Nyang'hwale districts' population was 807,619 and 148,320 respectively according to 2012 census (United Republic of Tanzania, 2012). The district projected population for 2011, 2012, 2013, 2014 and 2015 were obtained from the Districts' Planning, Monitoring and Statistics Departments.

IRS and ITNs coverage: The IRS and ITNs coverage data were obtained from the Districts' Health Departments. The number of ITNs consisted of the ITNs distributed to children under the age of five through children welfare clinics, pregnant women through antenatal clinics and ITNs distributed door to door freely. Local leaders were required to register the names of people in every household. The nets were distributed depending on the number of people per household. Before distributing nets, there were campaigns to encourage people to hang their nets once they are provided. It is assumed that the ITNs life of use is three years and one ITN on average protects two persons (Kamuliwo et al., 2013).

Data analysis: To determine the association between the burden of malaria, being the total of both clinically diagnosed and confirmed cases, and ITNs and IRS coverage, a Logistic regression was used. The IRS coverage rates were calculated as [households sprayed/ number of households in a given year] $x 100$, while IRS coverage rates were calculated as [households sprayed/number of households in a given year] $\mathrm{x} 100$. Below is the final fitted model used to calculate estimated probability of malaria cases.

Estimated probability of malaria cases = $\frac{\theta^{(2.5-3.0 x \text { ITNs coverage })}}{1+\theta^{(2.5-3.0 x \text { ITNs covarage }}}$

The data were cleaned in Microsoft Office Excel 2007 before analysis. All statistical analyses and graph production were performed by using STATA 12 (Stata Corporation, College Station, TX, USA). At 95\% confidence interval, a p-value less than 0.05 was statistically significant.

\section{RESULTS AND DISCUSSION}

Impact of the combined intervention with ITNs 
(a)

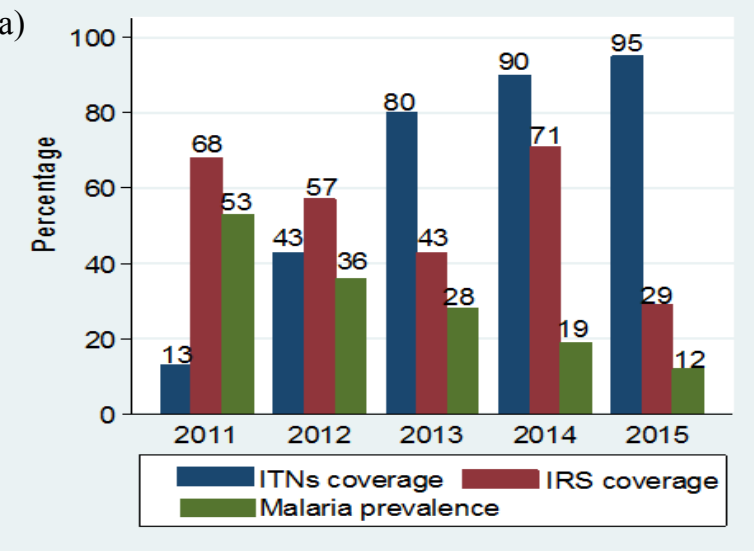

(b)

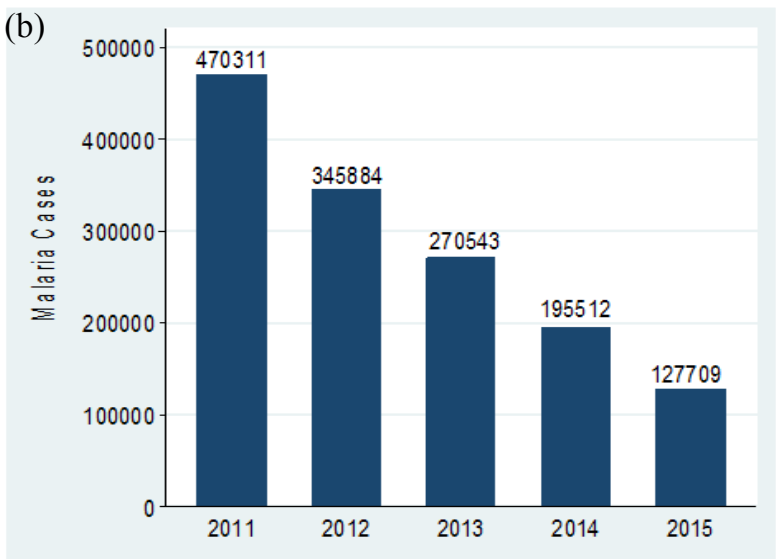

Fig. 2. (a) Effect of ITNs + IRS intervention on malaria prevalence and (b) malaria cases in Geita district from 2011 to 2015.

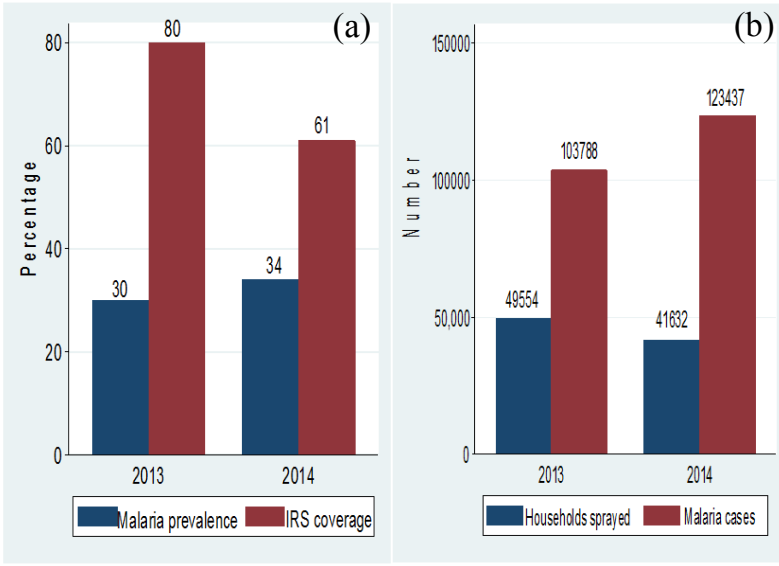

Fig. 3. Effect of IRS intervention on malaria prevalence and cases in Nyang'hwale district between 2013 and 2014. (a) IRS coverage and malaria prevalence (b) Households sprayed and malaria cases.

combined with IRS in Geita district and IRS alone in Nyang'hwale district on malaria prevalence: Percentage malaria cases and intervention coverage in Geita and Nyang'hwale districts are shown in figure 2 and 3. The insecticides for ITNs (Olyset Nets) and IRS were permethrin (WHO, 2014c; Graham, 2011) and lambda-cyhalothrin (http://www.rti.org/files/TVCSPSuccess-Geita-Tanzania) respectively. There was a reduction in malaria prevalence in Geita district, where the combined intervention of ITN and IRS was used from $53 \%(470,311)$ prevalence in 2011 to $12 \%$ $(127,709)$ prevalence in 2015 . There was a sharp decline in malaria prevalence between 2011 and 2012. Reduction in malaria prevalence remained somehow constant from 2012 to 2015 despite the sharp increase in the number of ITNs distributed (Fig. 2(a)). This was further reflected by the odds and the corresponding odds ratios of malaria cases from 2011 to 2015 (Table 1). However, there was an uneven IRS coverage from 2012 to 2015. On the other hand in Nyang'hwale district where only IRS was applied to control malaria transmission, malaria prevalence was $30 \%(103,788)$ and $34 \%(123,437)$, in 2013 and in 2014, respectively, while the number of households sprayed was $80 \%$ $(49,554)$ in 2013 and $61 \%(41,632)$ in 2014 (Fig. 3). In Nyang'hwale district where only IRS was conducted to control malaria vector population, there was an increase in malaria prevalence with a corresponding decline in IRS coverage (Fig.3a). These results indirectly provide evidence that IRS with a pyrethroid insecticide lambda cyhalothrin had an impact on malaria prevalence recorded in Nyang'hwale district because the decrease in IRS coverage was followed by an increase in malaria prevalence. Similarly, IRS with lambdacyhalothrin proved to be effective in reducing malaria transmission as indicated by earlier studies (Mashauri et al., 2013 and Raghavendra et al., 2011). Furthermore, in this study, it was revealed that the combined intervention of ITNs (pyrethroid) and IRS (pyrethroid) resulted in a reduction of malaria prevalence in Geita district (Fig. 2 (a)). These findings are in accordance with those of other studies conducted in Zambia and Botswana (Kamuliwo et al., 2013 and Simon et al., 2013) that reported a reduction in malaria prevalence, although the IRS insecticides were pirimiphos-methyl, an organophosphorus, and DDT, an organochlorine, respectively. The reduced malaria prevalence in Geita district may not all be attributed to the use of IRS and ITNs together. There has been a rapid increase in house construction in the district, and larger parts of these areas were marsh lands which have helped to destroy mosquitoes breeding sites leading to decreased mosquito population. Hence, environmental changes are likely to have an impact on malaria morbidity and mortality, in this case, due to a reduction in mosquito population (Mboera et al., 2013 and Simon et al., 2013).

Association between the interventions (IRS and ITNs) and malaria prevalence: The association between type of intervention and malaria prevalence was calculated using a Logistic regression model. Results of the association between malaria cases and IRS or ITN in the combined intervention are shown in Table 
Table 1. Odds of malaria cases and their corresponding Odds Ratios for the five years (2011- 2015)

\begin{tabular}{lccccc}
\hline & \multicolumn{5}{c}{ Year } \\
\cline { 2 - 5 } & $\mathbf{2 0 1 1}$ & $\mathbf{2 0 1 2}$ & $\mathbf{2 0 1 3}$ & $\mathbf{2 0 1 4}$ & $\mathbf{2 0 1 5}$ \\
\hline Odds & 1.15 & 0.57 & 0.38 & 0.24 & 0.14 \\
Odds Ratio & 1.00 & 0.50 & 0.33 & 0.21 & 0.12 \\
\hline
\end{tabular}

Table 2. Association between malaria cases and IRS or ITNs in the combined intervention

\begin{tabular}{lcccc}
\hline & \multicolumn{3}{c}{ Variables } \\
\cline { 2 - 5 } & OR & P-Value & [95\% Confidence Interval] \\
\hline IRS & 1.34 & 0.134 & $(0.643$ & $4.793)$ \\
ITNs & 0.05 & $<0.001$ & $(0.001$ & $0.002)$ \\
\hline
\end{tabular}

Table 3. Relationship between interventions and malaria cases.

\begin{tabular}{llll}
\hline & Malaria cases & IRS & ITN \\
\hline IRS & 0.16 & 1.00 & \\
ITNs & -0.53 & 0.20 & 1.00 \\
\hline
\end{tabular}

2. Insecticide-treated nets were found to have a significant $(p<0.001)$ association with reduced malaria cases while IRS was not $(p=0.134)$. If ITNs coverage were $50 \%, 80 \%$ and $100 \%$, malaria cases probabilities would be $0.73,0.52$ and 0.34 respectively. It was further observed that there was a negative relationship ( $\mathrm{r}$ $=-0.53$ ) between ITNs and malaria cases, meaning that as ITN coverage increased, malaria cases decreased. In addition, there was a very weak positive relationship $(\mathrm{r}=0.16)$ between IRS and malaria cases, which was far from being a perfect association (Table 3 ), a perfect association $r$ is close or equal to +1 or -1 . This implies that as IRS change there were no predictable changes on malaria cases. ITNs were found to have a significant association with reduced malaria cases while IRS was not (Table 2). The results may be due to increased ITNs coverage from 2011 to 2015 (Fig. 2(a)). These results are comparable to the study by Kamuliwo et al. (2013), which reported a significant association between ITNs and reduced malaria prevalence. Additionally, IRS protection is likely to be lower where ITNs coverage is at high levels (West et al., 2014 and WHO, 2014a). This is supported by a study which reported that there was no added benefit for combining ITNs and IRS in areas with a background of high ITN coverage (Corbel et al., 2012). Meanwhile, the association of ITNs with the reduced malaria cases in this study is contrary to a previous study which reported that IRS was associated with malaria cases reduction, but ITNs were not (Simon et $a l ., 2013)$. The difference in the findings between these two studies may be attributed to the different type of IRS insecticides and different coverage rates. How- ever, ITNs (pyrethroid) had been in use before IRS was introduced. Therefore there are possibilities that mosquitoes had developed resistance to pyrethroid which might be attributable to IRS lack of impact on malaria prevalence. If an insecticide of a particular mode of action is frequently used will lead to insecticide resistance (Insecticide Resistance Action Committee, 2011 and Graham, 2011) and if insecticides are to be used in both methods, it is important to use insecticides with different modes of action (Hemingway, 2014)

Difference in malaria burden between Geita district (ITNs + IRS) and Nyang'hwale district (IRS alone): A state epidemiological table feature [csi] was used to compare the impact of the two interventions on malaria prevalence between the two districts. A comparison between ITNs combined with IRS and IRS alone revealed a prevalence difference of $9 \%(p<0.001)$ (Table $4)$ and prevalence ratio was $0.73(p<0.001)$. These results show that the combined intervention had had an added benefit compared to IRS alone, but the average IRS coverage for the two years 2013 and 2014 was $57 \%$ and $71 \%$ in Geita and Nyang'hwale respectively. The low IRS coverage in Geita and Nyang'hwale districts may have contributed to the added benefit of the combined intervention. In order to generate protective effect against malaria transmission IRS coverage should at least be $80 \%$ (WHO, 2016).

\section{Conclusion}

It can be concluded from this study that malaria vector control by means of combined intervention in Geita district reduced malaria prevalence while in Nyang'hwale district where IRS alone was applied there was an increase in malaria prevalence accompanied by a decline in IRS coverage. A Logistic regression model showed that in the combined intervention, ITNs had a significant effect $(p<0.001)$ but IRS did not $(p=0.134)$ and if the ITN coverage were $50 \%, 80 \%$ and $100 \%$, estimated probability of finding malaria cases in Geita district would be $0.73,0.52$ and 0.34 respectively.

\section{ACKNOWLEDGEMENTS}

The INTRA-ACP Mobility Scholarship Scheme is acknowledged for funding the study. We also thank Professor Dominic M. Kambarage and Dr. Martin C.

Table 4. Difference in impact on malaria prevalence between ITNs + IRS and IRS alone.

\begin{tabular}{llllll}
\hline & ITNS + IRS & IRS alone & P-Value & [95\% Confidence Interval] \\
\hline Prevalence & $0.23(23 \%)$ & $0.32(32 \%)$ & & & \\
& Point estimation & & & & \\
Prevalence difference & -0.09 & & 0.00 & $(-0.087$ & $-0.085)$ \\
Prevalence ratio & 0.73 & 0.00 & $(0.728$ & $0.735)$ \\
\hline
\end{tabular}


Simuunza for coordinating the project.

\section{REFERENCES}

CDC (2014). Fighting malaria and saving lives; President's Malaria Initiative., Center for Disease Control and Prevention - CDC. Washington.

Corbel V., Akogbeto, M., Damien, G.B., Djenontin, A., Chandre, F., Rogier, C., Moiroux, N., Chabi, J., Banganna, B., Padonou, G.G. and Henry, M. (2012). Combination of malaria vector control interventions in pyrethroid resistance area in Benin: a cluster randomized controlled trial. The Lancet Infectious Diseases, 12 (8): 617-626. (Accessed on 09/04/2016.

Graham, M. (2011). Integrated vector management: controlling vectors of malaria and other insect vector-borne diseases, First Edition, 163 pp. West Sussex, UK, John Wiley \& Sons, Ltd.

Hemingway, J. (2014). The role of vector control in stopping the transmission of malaria; threats and opportunities. Philosophical Transaction of Royal Society, B.369: 20130431

Insecticide Resistance Action Committee. (2011). A manual on prevention and management of insecticides resistance in vectors of public health importance. Second Edition

Jo L. and Kleinschmidt, I. (2013). Combining malaria vector control interventions: some trial design. Pathogens and Global Health, 107(1): 1-4

Kamuliwo M., Chanda, E., Haque, U., Mwanza- Ingwe, M., Sikaala, C., Katebe- Sakala, C., Mukonka, V.M., Norris, D.E., Smith, D.L., G. E. Glass, G.E. and M.J.William, M.J. (2013). The changing burden of malaria and association with vector control interventions in Zambia using district - level surveillance data, 20062011. Malaria Journal, 12: 437

Kigozi R., Baxi, S.M., Gasasira, A., Sserwanga, A., Kakeeto, S., Nasr, S., Rubahika, D., Dissanayake, G., Kamya, M.R., Filler, S. and Dorsey. G. (2012). Indoor residual spraying of insecticide and malaria in high transmission intensity area of Uganda. PLoS One. 7(8): e42857

Kim D., Kristen, F. and Randall, K. (2012). Reduction of malaria prevalence by indoor residual spraying: a metaregression analysis. The American Journal of Tropical Medicine and Hygiene, 87 (1): 117- 124.

Kleinschmidt I., Schwabe, C., Shiva, M., Segura, J.L., Sima, V., Mabunda ,S.J.A. and Coleman M. (2009). Combining indoor residual spraying and insecticide-treated nets interventions. American Journal of Tropical Medicine and Hygiene, 81(3): 519-524

Mashauri F.M., Kinung'hi, S. M., Kaatano, G. M., _Magesa, S. M., _Kishamawe, C., Mwanga, J. R., Nnko, S. E., Malima, R. C., Mero, C. N. and Mboera L. E. G. (2013). Impact of indoor residual spraying of lambdacyhalothrin on malaria prevalence and anemia in an epidemic-prone district of Muleba, North-Western Tanzania. The American Journal of Tropical Medicine and Hygiene, 88 (5): 841-849

Mboera L.E.G., Mazigo, H. D., Rumisha S F. and Randall K. A. (2013). Towards malaria elimination and implication for vector control, disease management and liveli- hoods in Tanzania. Malaria World Journal, 4:19.

Moyer J. D. and Graham E. (2012). Malaria no more expectations for eradication. African Futures Brief; International Futures No. 5

Okonko, I.O, Soleye, F.A., Amusan, T.A., Ogun, A.A., Udeze, O.A., Nkang, I.O.A., Ejembi, J. and Faleye, C.O.T. (2009). Prevalence of malaria plasmodium in Abekuta, Nigeria. Malaysian Journal of Microbiology, 5(2): 113-118.

Okumu O.F. and Moore S.J. (2011). Combining indoor residual spraying and insecticide-treated nets for malaria control in Africa: A review of possible outcomes and outline of suggestions for the future. Malaria Journal, 10:208

PMI (2014). Tanzania, malaria operational FY 2014. http:// www.pmi.gov/docs/default-source/default-documentlibray/malaria-operational-plans/fy14/ tanzania_mop_fy14.pdf?sfvrsn=12.President's Malaria Initiative. (Accessed 08/04/2016)

Raghavendra K., Ghosh, S.K., Eapen, A., Tiwari, S.N., Satyanarayan, T.S., Ravindran, J., Sreehari, U. and Dash, A.P. ( 2011). (ICON 10 CS) Field evaluation of lambda-cyhalothrin (ICON $10 \mathrm{CS}$ ) indoor residual spraying against Anopheles culicifacies in India." Journal of Vector Borne Diseases, 48:18- 26

Recognizing mutual benefits through public-private partnerships in Tanzania. The Geita experience; success story. http;//www.rti.org/files/TVCSP-Success-GeitaTanzania. (Accessed 15.08.2015).

Simon C., Moakofhi, K., Mosweunyane, T., Jibril, H.B., Nkomo, B., Motlaleng, M., Ntebela, D.S., Chanda, E. and Haque, U. (2013). Malaria control in Botswana, 2008-2012: The path towards elimination" Malaria Journal, 12 (1): 458

United Republic of Tanzania (2012). Tanzania national census.

United Republic of Tanzania. (2013). Tanzania HIV/AIDS and malaria indicator survey 2011-12: key findings. Dar - es- Salaam, Tanzania.

West P.A., Protopopoff, N., Wright, A., Kivaju, Z., Tigererwa, R., Mosha, F. W., Kisinza, W., Rowland, M. and Kleinschmidt I. (2014). Indoor residual spraying in combination with insecticide-treated nets compared to insecticide-treated nets alone for protection against malaria: a cluster randomised trial in Tanzania. PLoS Medicine, 11(4), e1001630.

WHO (2016). Malaria fact sheet. http:/www.who.int/ mediacentre/factsheets/fs094/en/ (accessed on $09 / 04 / 2016)$

WHO (2014c). Recommended long-lasting insecticidal nets. http;//www.who.int/whopes/quality/newspecif/en/ (Accessed on 15/04/2016)

WHO (2015). World Malaria Programme; World Malaria Report

WHO (2014b). Review of current evidence on combining indoor residual spraying and long-lasting insecticidal nets. Malaria policy advisory committee meeting.http:// www.who.int/malaria/mpac/background-combining-irs1linsmar2014.pdf. (accessed on 09/04/2016)

WHO (2014d). Review of current evidence on combining indoor residual spraying and long-lasting insecticidal nets. Malaria policy advisory committee meeting. 